NOTAS SOBRE LA RESPONSABILIDAD DE LAS PERSONAS JURİDICAS POR LOS DELITOS EN EL SISTEMA ITALIANO, CON PARTICULAR REFERENCIA A LA DENOMINADA "CULPA DE ORGANIZACIÓN"

NOTES ABOUT "EX CRIMINE" LIABILITY OF LEGAL PERSONS IN THE ITALIAN SYSTEM, WITH PARTICULAR REFERENCE TO THE SO-CALLED "ORGANIZATIONAL FAULT"

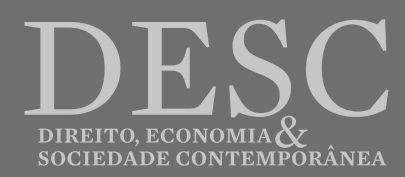




\title{
NOTAS SOBRE LA RESPONSABILIDAD DE LAS PERSONAS JURÍDICAS POR LOS DELITOS EN EL SISTEMA ITALIANO, CON PARTICULAR REFERENCIA A LA DENOMINADA “CULPA DE ORGANIZACIÓN"*
}

\author{
NOTES ABOUT "EX CRIMINE” LIABILITY OF LEGAL \\ PERSONS IN THE ITALIAN SYSTEM, WITH PARTICULAR \\ REFERENCE TO THE SO-CALLED \\ "ORGANIZATIONAL FAULT"
}

\begin{abstract}
Resumo: O texto aborda a responsabilidade penal das pessoas jurídicas no direito italiano. Para isso, adota novos critérios de imputação, tratando a conduta delitiva como "defeito organizacional" que favorece a prática da conduta criminosa. Sob essa perspectiva, a imputação do crime à pessoa jurídica pode ser feita por cinco níveis de ligação: i) a pessoa jurídica ser a beneficiária do crime; ii) ter sido o crime cometido por falha de supervisão; iii) o crime ter ocorrido por falha organizacional; iv) ausência de mecanismos de prevenção de crimes; v) ter sido o crime cometido por uma "evasão não fraudulenta" do modelo organizacional.
\end{abstract}

Palavras-chave: responsabilidade penal, pessoa jurídica, critérios de imputação.

\begin{abstract}
The text addresses the 'ex crimine' liability of legal entities in the Italian law. For this, it adopts new imputation criteria, that are based on the concept of 'organizational defect' to guarantee the full belonging of the offence to the legal entity, respecting the principles of personality and culpability.

From this perspective, the imputation of the crime to the legal entity can be done by five levels of connection: i) the legal entity is the beneficiary of the crime; ii) the offense has been committed by a subject in an apical or subordinate position in the entity; iii) the crime occurred because of organizational failure; iv) before the commission of the offence, the legal entity has not adopted adequate mechanisms for the prevention of crimes of the same kind; $v$ ) the crime has been committed by a 'fraudulent evasion' of the organizational model.
\end{abstract}

Keywords: 'ex crimine' liability, legal entity, imputation criteria, organizational fault 


\section{Introducción}

Entre los diferentes países de la Unión Europea, Italia ha introducido una regulación de la responsabilidad de las personas jurídicas por la comisión de delito que está muy avanzada, hasta el punto de servir de inspiración de otras leyes europeas y no europeas ${ }^{1}$ y que ha sido considerado por parte de la doctrina como un ejemplo a seguir ${ }^{2}$. Se trata de un modelo que se basa la imputación de la responsabilidad en el "defecto de organización", por las razones que a continuación señalaré.

El decreto legislativo n. 231, de 8 de junio introdujo una responsabilidad formalmente "administrativa"” de las entidades colectivas, que encuentra su premisa en la comisión de un delito, por una persona relacionada con la persona jurídica, en el interés o en beneficio de la misma; responsabilidad de la entidad que se suma, sin sustituirla, a la del individuo autor del delito, de acuerdo con un principio de autonomía de las dos esferas de responsabilidad ${ }^{4}$. Se trata, en otras palabras, de una responsabilidad "acumulativa" de la entidad, y no alternativa, a la de la persona física ${ }^{5}$. Las responsabilidades del individuo y de la entidad colectiva se establecen en el mismo juicio penal, garantizando a la persona jurídica todos los derechos procesales de que se disponen para el caso de personas físicas.

\section{El debate sobre la naturaleza de la responsabilidad de las entidades colectivas por la comisión de delitos}

En la doctrina italiana se ha desarrollado un amplio debate sobre la "naturaleza" de

\footnotetext{
1 * Este artículo fue publicado en España, en la Revista General de Derecho Penal, № 23 Mayo 2015 y en Perù, en Actualidad penal, Vol. 17, Noviembre 2015, pp. 124-143. Este artículo, bajo el título "Responsabilidad penal de las personas jurídicas en Italia”, también fue publicado en Colombia, en Derecho Penal Contemporáneo, Vol. 53, Octubre-Diciembre 2015, pp. 169194.

Por ejemplo, entre la legislación europea, vid. la "Bribery Act” de 2010, el artículo 102 del Código penal suizo o el reciente Anteproyecto de reforma del Código penal español (Proyecto de ley orgánica por la que se modifica la ley orgánica 10/1995, de 23 de noviembre, del Código penal), que propone enmendar el artículo 31 bis del Código penal español. Vid., igualmente, la ley chilena (Ley n. 20.393 de 2.12.2009) y el Anteproyecto de ley que regula la responsabilidad penal de las personas juridicas en los delitos de corrupción en Perú, presentado por la CAN el 9.12.2013.

2 En la doctrina española, en este sentido, A. Nieto Martín, El artículo 31 bis del Código Penal y las reformas sin estreno, in Diario la ley, 2014, n. 8248, p. 2, aplaude el modelo italiano de atribución de la responsabilidad a las personas jurídicas por los delitos. Además en la doctrina anglosajona, J. GoBert, M. Punch, Rethinking Corporate Crime, Cambridge, 2003, p. 108 ss., al comentar sobre nuestro sistema de imputación de la responsabilidad a las personas jurídicas, hacen referencia a las "Lessons from Italy"; y, antes aún, J. GoBert, E. MugnaI, Coping with Corporate Criminality - Some Lessons from Italy, in Criminal Law Review, 2002, p. 623 ss. Para un análisis comparativo en profundidad de los modelos de imputación contenidos en los ordenamientos jurídicos de los 28 países de la Unión Europea, se puede hacer referencia a AA.VV., Corporate criminal liability and compliance programs, Vol. I, Liability 'ex crimine' of legal entities in Member States, editado por A. Fiorella, Nápoles, 2012.

3 Sobre el debate sobre la naturaleza de esta responsabilidad en la doctrina y en la jurisprudencia italiana, vide infra, n. 2.

4 Sobre los efectos del principio de autonomía de las dos esferas de responsabilidad para la imputación de la responsabilidad a las entidades colectivas por un hecho delictivo, vid. D. Pulitanò, Responsabilità amministrativa per i reati delle persone giuridiche, in Enc. dir., 2002, VI agg., p. 962 s. En la jurisprudencia, Cass. pen., Sez. VI, 6.2.2009, n. 19764, en Guida dir., 2009 , n. 26 , p. 82.

5 La responsabilidad de la entidad será exigible siempre que se constate la comisión de un delito, aun cuando la concreta persona física responsable no haya sido individualizada o no haya sido posible dirigir el procedimiento contra ella, por ejemplo, porque no sea imputable, según el art. 8 d. lgs. 231/2001.
} 
la responsabilidad de las entidades colectivas por delitos. Formalmente, el legislador italiano la denominó "responsabilidad administrativa", si bien es de la doctrina mayoritaria la que la concibe como una forma de responsabilidad "penal"6 o por lo menos "cuasi-penal", cuya disciplina parecería articular un "subsistema" autónomo en el sistema penal".

La naturaleza "penal" o "cuasi-penal" de la responsabilidad se puede derivar de la estrecha relación entre el delito y la imputación de la responsabilidad a la entidad colectiva; de las sanciones sumamente restrictivas para la entidad responsable, que incluyen la suspensión de sus actividades; además de que la responsabilidad de la entidad se establece en el marco del proceso penal ante un juez penal.

Los que niegan la naturaleza "penal" o "cuasi-penal" de la responsabilidad de las entidades colectivas justifican su opinión, desde un punto de vista formal, en el hecho de que el d. lgs. 231/2001 se refiere expresamente a la responsabilidad "administrativa" de las mismas entidades y, desde un punto de vista sustantivo, invocan el conocido principio de "societas delinquere non potest" a la luz de los principios constitucionales. Se refieren al

6 A favor de una naturaleza esencialmente "penal”, G. Amarelli, Mito giuridico ed evoluzione della realtà: il crollo del principio societas delinquere non potest, in Riv. trim. dir. pen. econ., 2003, p. 967 ss.; A. CARMONA, Premesse a un corso di diritto penale dell'economia, Padova, 2002, p. 208; G. DE Simone, La responsabilità da reato della societas nell'ottica dei principi costituzionali, in AA.VV., La responsabilità da reato degli enti collettivi: a dieci anni dal d.lgs. n. 231/2001. Problemi applicativi e prospettive di riforma, editado por V. Mongillo, A.M. Stile, G. Stile, Nápoles, 2013, p. 263; G. De Vero, La responsabilità penale delle persone giuridiche, in Trattato di diritto penale, diretto da C.F. Grosso, T. PAdovani, A. PAGLiaro, Milano, 2008, p. 322 ss.; R. GUERRINI, La responsabilità da reato degli enti. Sanzioni e loro natura, Milano, 2006, p. 233 s.; V. Maiello, La natura (formalmente amministrativa, ma sostanzialmente penale) della responsabilità degli enti nel d. lgs. n. 231/2001: una «truffa delle etichette» davvero innocua?, in Riv. trim. dir. pen. econ., 2002, p. 899 ss.; A. MANNA, La c.d. responsabilità amministrativa delle persone giuridiche: il punto di vista del penalista, in Cass. pen., 2003, p. 1103 ss.; E. Musco, Le imprese a scuola di responsabilità tra pene pecuniarie e misure interdittive, in Dir. e giust., 2001, n. 23, p. 8 s.; T. PADOVANI, Il nome dei principi e il principio dei nomi: la responsabilità "amministrativa" delle persone giuridiche, in AA.VV., La responsabilità degli enti: un nuovo modello di giustizia "punitiva", editado por G. DE FrancEsCo, Torino, 2004, p. 16 s.; C.E. PALiero, Il d. lgs. n. 231/2001: da ora in poi, societas delinquere (et puniri) potest, in Corr. Giur., 2001, n. 7, p. 845 ss.; P. Patrono, Verso la soggettività penale di società ed enti, in Riv. trim. dir. pen. econ., 2002, n. 1-2, p. 186 ss.

7 En este sentido, se refieren a una responsabilidad "cuasi-penal" ("para-penale"): A. Fiorella, Responsabilità da reato degli enti collettivi, in Dizionario di diritto pubblico, vol. V, dirigido por S. Cassese, Milano, 2006, p. 5101; N. SelvagGi, L'interesse dell'ente collettivo quale criterio di ascrizione della responsabilità da reato, Nápoles, 2006, p. 55 s.; A.S. VALENZANO, Responsabilità degli enti e criminalità transnazionale anche nella prospettiva dei rapporti tra l'Unione Europea e le Nazioni Unite, in AA.VV., Rapporti tra Unione Europea e organizzazioni internazionali, editado por E. PFöstL, Roma, 2009 , p. 239. Se refiere a un "tertium genus" de responsabilidad de las entidades colectivas el Informe del Gobierno al decreto (Relazione al d. lgs. 231/2001, in www.confindustria.it) y en la doctrina: O. Di Giovine, La responsabilità degli enti: lineamenti di un nuovo modello di illecito punitivo, in AA.VV., Diritto e impresa: un rapporto controverso, editado por A. MANNA, Milán, 2004, p. 435; G. FlORA, Le sanzioni punitive nei confronti delle persone giuridiche: un esempio di «metamorfosi» della sanzione penale?, in Dir. pen. proc., 2003, p. 1398 ss.; G. ForTI, Sulla definizione della colpa nel progetto di riforma del codice penale, in C. DE Maglie, S. Seminara, La riforma del codice penale. La parte generale, Actas de la Conferencia de Pavia, 10-12 maggio 2001, Milán, 2002; L. Stortoni, D. TAssinari, La responsabilità degli enti: quale natura? Quali soggetti?, in Indice pen., 2006, p. 9 ss. En la jurisprudencia, en este sentido, Cass. pen., Sez. VI, 16.7.2010, n. 27735, in www.rivista231.it; Cass. pen., Sez. VI, 17.9.2009, n. 36083, in www. rivista231.it.

8 El d. lgs. n. 231/2001 cumple con los siguientes principios fundamentales en materia de derecho penal: el principio de legalidad, ya previsto por el artículo 25 de la Constitución y en los artículos 1 y 199 del Código penal, y también el principio de no retroactividad de la ley, sin perjuicio de la retroactividad de la ley más favorable a la entidad colectiva (artículos 2 y 3 del decreto). Además, con referencia específica al principio de territorialidad que normalmente rige la jurisdicción penal, debe indicarse que el d. lgs. n. 231/01 proporciona una excepción a este principio al afirmar que las entidades que tienen en el territorio del Estado italiano la sede central pueden ser responsables en relación a los delitos cometidos en otros países, siempre que no ejerza su jurisdicción el Estado del lugar donde se cometió el delito, en los casos y en las condiciones previstas en el código penal (artículos 7, 8, 9 y 10 del decreto). 
principio de "culpabilidad", garantizado por el articulo 27 de la Constitución italiana, con el cual entraría en conflicto la responsabilidad "penal" de las entidades colectivas.

Más en particular, el artículo 27 de la Constitución establece, en primer lugar, el principio de "personalidad de la responsabilidad penal" ("personalità della responsabilità penale”), según el cual "la responsabilidad penal es personal". Lo dispuesto en este precepto se ha interpretado por la doctrina en el sentido de que nadie puede ser sancionado si no que ha cometido un hecho "propio", es decir, se trata de una responsabilidad "personal" y subjetiva que requiere dolo o culpa.

En esencia, desde el principio de "personalidad de la responsabilidad penal" se pueden derivar los siguientes corolarios: primero, la responsabilidad penal requiere que una persona haya cometido un "hecho propio", es decir, un hecho que es objetivamente imputable a él por un nexo de causalidad entre su conducta y el resultado que del mismo se deriva. Por lo tanto, este principio excluye que cualquier forma de responsabilidad penal "por el hecho de otra persona" ("per fatto altrui"), es decir por un hecho cometido por otros sujetos.

En segundo lugar, la responsabilidad penal exige que una persona haya cometido un "hecho culpable", es decir un hecho cometido con dolo, o con culpa en los casos expresamente previstos por la ley, y por lo tanto que sea "reprochable" ("rimproverabile") (concepción "normativa" de la culpabilidad). Este principio excluye,, al menos en principio, que en nuestro sistema penal se acepten formas de "responsabilidad objetiva", que requieren tan sólo un nexo de causalidad entre la conducta y el hecho dañoso o peligroso, sin necesidad de verificar un elemento psicológico (dolo o culpa), tal como se admite en el derecho civil.

En mi opinión, la cuestión fundamental para admitir la responsabilidad "penal" o por lo menos "cuasi-penal" de las entidades colectivas es que, desde el punto de vista de las garantías sustantivas y procesales, los principios constitucionales establecidos para el individuo acusado de un delito en cualquier caso deben ser garantizados, mutatis mutandis, aún para la empresa acusada de una infracción por un hecho delictivo. A continuación explicaré cómo se garantizan los principios constitucionales y, en particular, los principios de "personalidad de la responsabilidad penal" y de "culpabilidad" por las normas contenidas en el d. lgs. n. 231/2001.

\section{Introducción al análisis de los criterios de imputación de la responsabilidad a las entidades colectivas por un hecho delictivo}

En cuanto a la imputación de la responsabilidad, es sabido que nuestro sistema penal se ha estructurado tradicionalmente sobre el principio de responsabilidad "penal" exclusivamente de las personas físicas. En relación al mismo, se construyeron dos criterios fundamentales de imputación de la responsabilidad: uno causal y otro psicológico (dolo o 
culpa). Ahora bien, si se hace una interpretación estricta, el elemento subjetivo del delito no se puede imputar a la persona jurídica, porque ésta no puede expresar una "voluntad" e en el sentido común de la voluntad propia de la persona física.

Para superar estos problemas, el legislador italiano ha tratado de garantizar los principios de "personalidad de la responsabilidad penal" y de "culpabilidad" a través de la elaboración de un nuevo sistema de criterios de imputación previstos en el d. lgs. n. 231/2001 y que establecen un estrecho vínculo entre el delito y la entidad colectiva para demostrar la "pertenencia" ( "appartenenza”) del primero a la segunda.

Como indicaré después, nuestro legislador ha estructurado un sistema de imputación del hecho delictivo a la entidad colectiva sobre la base del ejercicio de poder inapropiado de la organización que se refleja en un "defecto de organización" o en una "política de empresa", que respectivamente permite o incluso favorece para la comisión de delitos. En esencia, la responsabilidad se atribuye a la entidad colectiva cuando existe una estrecha "conexión" entre el delito cometido y su "defecto de organización", sin la cual no se puede atribuir el "reproche" a la entidad.

En otras palabras, el legislador ha ideado un complejo mecanismo para la atribución de la responsabilidad a las entidades colectivas por un hecho delictivo, esencialmente haciendo eco de la lógica que subyace a la atribución por la "omisión de impedir delitos" ("omesso impedimento del reato" "), estableciendo cinco niveles de "pertenencia" ( "appartenenza" 10 ) del hecho delictivo a la persona jurídica. Sin olvidar el rol que, por supuesto, la acción de la entidad colectiva puede tener en la contribución a la comisión del delito, por ejemplo, cuando la conducta activa manifiesta una opción de "política de empresa", que de alguna manera ha instigado o dado lugar a la comisión del delito.

Con referencia a los cinco niveles de "pertenencia", el delito se puede imputar a la entidad colectiva ${ }^{11}$, si bien como infracción administrativa, si es:

1) diseñado genéticamente para lograr el "interés" de la propia entidad, que, por lo tanto, es la beneficiaria del delito (pertenencia “por destinación”) (appartenenza "per destinazione");

\footnotetext{
9 En el sistema italiano, la atribución de la responsabilidad a una persona fisica por no haber impedido un delito está regulada en el art. 40 párrafo 2 c.p., que establece que "no impedir un evento, que se tiene el deber jurídico de evitar, equivale a causarlo". Para un análisis del complejo debate doctrinal sobre este tema, vid. A.S. VALENZANo, Modelli organizzativi e organismo di vigilanza nel sistema della responsabilità dell'ente per fatti di reato, Roma, 2007, p. 99 ss.

10 Para un análisis en profundidad de los cinco niveles, A. Fiorella, Responsabilità da reato degli enti collettivi, cit., p. 5102 ss.

11 Los destinatarios de la disciplina son, según el art. 1 del d. lgs. 231/2001, las entidades con personalidad jurídica, las sociedades y también las asociaciones sin personalidad jurídica, con la excepción del Estado, los gobiernos locales, las otras entidades públicas no económicas, así como las entidades que ejercen funciones constitucionales. Para un análisis de estos temas en la doctrina y en la jurisprudencia italiana, vid. S. MASsI, 'Veste formale'e 'corpo organizzativo' nella definizione del soggetto responsabile per l'illecito da reato, Nápoles, 2012.
} 
2) cometido por el "ápice" o éste lo facilita al incumplir el deber de supervisión o dirección (pertenencia "por la posición jerárquica del sujeto activo") (appartenenza "per la significativa posizione del soggetto attivo”).

La disciplina italiana se completa también con los siguientes niveles adicionales de imputación, que podrían resumirse en la idea de que el delito debe ser el resultado de un "defecto de organización". Por lo tanto, el delito debe ser:

3) causado "en concreto" por un "defecto de organización" (pertenencia "por la derivación del hecho de un defecto de organización") (appartenenza "per la derivazione del fatto da una lacuna dell'organizzazione”);

4) posible gracias a la falta de un modelo de organización "normalmente adecuado" para la prevención de delitos de la misma naturaleza respecto del que se ha cometido (pertenencia "por la derivación del riesgo no permitido de la empresa" o por la llamada "culpa de organización") (appartenenza "per la derivazione dal rischio non-permesso d'impresa" ovvero per la c.d. "colpa di organizzazione”);

5) cometido por una "elusión no fraudulenta" del modelo organizativo por parte del autor ápical del delito (pertenencia "por la elusión no fraudulenta del modelo organizzativo") (appartenenza "per la non-fraudolenta elusione del modello organizzativo”).

\section{El criterio del interés de la entidad colectiva}

El primer criterio objetivo de imputación de la responsabilidad a la entidad colectiva se encuentra en el hecho de que el delito se haya cometido "en interés" ("nell'interesse") o "en beneficio" ("a vantaggio") de la entidad colectiva.

La diferencia entre los conceptos de interés y beneficio se basa sustancialmente en el momento de su realización, ya que mientras el interés motiva la conducta criminal y, por lo tanto, se debe establecer "ex ante", por el contrario, el beneficio se establece "ex post"12.

En mi opinión, se debe tener cuidado al atribuir un significado correcto a éste último, ya que todos los criterios de imputación deben establecer una "conexión" esencial del hecho delictivo con la entidad colectiva y, por lo tanto, se debe identificar una conducta que, de alguna manera, sea "genéticamente" diseñada para lograr el interés de la entitad, por su unívoca dirección objetiva, y que coincida con la intención del individuo.

Queda por determinar si cualquier conducta que pueda ser cometida con un interés divergente o incompatible con el de la entidad colectiva, puede de hecho producir un beneficio para la misma entidad, como por ejemplo de naturaleza económica, y servir, por tanto, de base para la imputación de responsabilidad. En otras palabras, la realización de una ventaja

12 En este sentido se expresa el Informe del Gobierno al decreto (Relazione al d. lgs. n. 231/2001, cit.). 
(un mero beneficio) que puede ser de carácter ocasional, según una correcta interpretación de la norma, no debería fundamentar la responsabilidad de la entidad por el hecho delictivo que se hubiese cometido.

En conclusión, la correcta interpretación del decreto, de conformidad con el principio constitucional de "personalidad de la responsabilidad penal", requiere considerar la fórmula de la ley como un "endíadis", como si la ley fuera a decir que la responsabilidad debe imputarse a la entidad colectiva por una conducta que, en todos los casos, sea cometida en el interés de la misma, se produzca o no una ventaja para la misma. El beneficio no puede, por tanto, ser considerado como un criterio en sí mismo suficiente para conectar el hecho delictivo a la entidad colectiva ${ }^{13}$.

Esta interpretación viene confirmada por una atenta lectura del decreto (art. 5, párrafo 2), donde se establece que la entidad no es responsable si el individuo actuó "en propio interés exclusivo o de terceros" ("nell'interesse esclusivo proprio o di terzi"), lo que significa que el beneficio de la entidad por sí solo no es suficiente para imputar la responsabilidad a la misma y, por tanto, no constituye un criterio autónomo de imputación de la responsabilidad.

Sin embargo, es necesario tener en cuenta que la Corte Suprema es bastante oscilante sobre este tema, ya que ha considerado a veces el criterio del beneficio ("ex post") como "alternativo" a el de interés ("ex ante"), y por lo tanto por sí solo suficiente para atribuir la responsabilidad a la entidad por el hecho delictivo que sea comitido ${ }^{14}$.

\section{La posición jerárquica del sujeto activo}

El segundo criterio objetivo de imputación de la responsabilidad a la entidad colectiva se refiere al autor del delito que, en virtud del art. 5 del d. lgs. n. 231/2001, debe estar en una posición significativa, como "apical" o sujeto "subordinado". Para ser imputable a la entidad colectiva, el delito debe ser cometido por el "ápice” o facilitado por éste al no haber cumplido con sus deberes de supervisión o dirección.

El legislador define las dos posiciones subjetivas, que revisten importantes diferencias, como a continuación indicaré.

Los "apicales" se definen como las personas que tienen funciones de representación, administración o dirección de la entidad o de una de sus unidades de organización con autonomía financiera y funcional, así como las personas que ejercen, también de facto, la

13 Para más detalles, así como para un análisis de la jurisprudencia sobre este tema, N. SelvaGgi, L'interesse dell'ente collettivo quale criterio di ascrizione della responsabilità da reato, cit., p. 23 ss.

14 En particular, se hace referencia a Cass. pen., Sez. II, 30.1.2006, n. 3615, in Riv. pen., 2006, p. 615; por el contrario, Cass. pen., Sez. VI, 23.6.2006, n. 32626, in Cass. pen., 2007, p. 91, que excluye la posibilidad de que un beneficio "incidental" sea suficiente para imputar la responsabilidad a la persona juridica. 
gestión y control de la misma entidad. Los "subordinados", sin embargo, se definen como aquellas personas que están sujetas a la dirección o supervisión de uno de los "ápices".

Es importante enfatizar que, con el fin de garantizar una estrecha conexión entre el delito y la entidad colectiva a la luz del principio de "personalidad de la responsabilidad penal", en mi opinión, es necesario que estas personas estén "inmediatamente subordinadas" a la dirección o supervisión de un líder, es decir que no se encuentren en una posición lejana respecto a éste en la cadena de mando (jerarquía) y, por lo tanto, se encuentren en una posición elevada desde el punto de vista funcional. Por esta razón, parte de la doctrina se refiere a la necesaria posición "para-apical"15 de la persona "subordinada" a la dirección o supervisión de uno de los "ápices", porque de lo contrario se puede correr el riesgo de imputar a la entidad colectiva una conducta criminal que no debería ser imputada a la misma, porque se trata de una conducta que está muy lejos del centro de decisión, es decir, del centro de producción de la "voluntad" de la misma entidad.

\section{El nexo de causalidad entre el "defecto de organización" y el hecho delictivo}

El d. lgs. 231/2001 ha seguido la experiencia estadounidense para consolidar mejor la imputación del hecho delictivo a la entidad, enfatizando la idea de la “organización en general"16.

Por lo tanto, el tercer criterio de imputación de la responsabilidad a la entidad colectiva consiste en una relación de causalidad entre el "defecto de la organización" y el hecho delictivo, en el sentido de que el delito debe haber sido causado "en concreto" por una "falta de organización" (“organización defectuosa") o en cualquier caso por la "política de empresa".

En mi opinión, dada la compleja estructura de la infracción que se atribuye a la entidad colectiva, el "defecto de organización" puede ser considerado como la "conducta" imputable a la misma entidad, mientras que el delito cometido puede considerarse como el "resultado" de la infracción; resultado que, según los principios generales del derecho penal, debe ser causado concretamente por la conducta a través del nexo causal.

Esta interpretación de la estructura de la infracción de la entidad colectiva viene confirmada por algunas disposiciones del d. lgs. n. 231/2001. El art. 17, apartado 1, letra b), del decreto se refiere a "las deficiencias organizativas que han llevado al delito" ("le carenze organizzative che hanno determinato il reato"), aunque con referencia al comportamiento post-

15 A. Fiorella, Responsabilità da reato degli enti collettivi, cit., p. 5103; M. MAsucci, Infedeltà patrimoniale e offesa al patrimonio nella disciplina penale dei gruppi di società, Nápoles, 2006, p. 445 ss.; N. PIsANI, La struttura dell'illecito e i criteri di imputazione, in AA.VV., La responsabilità da reato degli enti, vol. X, editado por A. D’Avirro e A. Di Amato, Padova, 2009, p. 93 s. Para más detalles sobre este tema, también en una perspectiva comparada, se puede hacer referencia a A.S. VALENZANO, 'Triggering persons': the role of the offender in 'ex crimine' liability of legal entities. A comparative study on Member States' legal systems, in AA.VV., Corporate criminal liability and compliance programs, Vol. II, Towards a common model in the European Union, editado por A. Fiorella, Nápoles, 2012, p. 213 ss.

16 En este sentido se expresa el Informe del Gobierno al decreto (Relazione al d. lgs. n. 231/2001, cit.). 
factum de la entidad para eliminar estas deficiencias. Mientras el art. 7, apartado 1, del decreto establece que la entidad colectiva es responsable sólo si la comisión del delito por la persona "sometida" ha "sido posible" ("è stata resa possibile") por haberse incumplido los deberes de dirección o supervisión por parte de uno de los "ápices” de la misma entidad, es decir, porque se ha producido un "defecto de organización" que ha llevado a la comisión del delito.

\section{La denominada "culpa de organización" por ser inadecuado el modelo de prevención de delitos. La exención de responsabilidad en casos de entidades colectivas correctamente organizadas}

Nuestro sistema equilibra instrumentos represivos con otros de carácter preventivo, incentivando a las entidades colectivas a organizarse adecuadamente para prevenir la comisión de los delitos (también en referencia a la filosofía del llamado "carrot and stick approach" anglosajón ${ }^{17}$ ). La idea básica de la disciplina italiana, inspirada en el sistema federal estadounidense ${ }^{18}$, es la de la "prevención" de los delitos, en el sentido de que a través de la implantación del sistema de la responsabilidad de las entidades por delitos se intenta estimularlas para que se organicen "virtuosamente".

En mi opinión, la lógica de la prevención es la que puede producir los mejores resultados en la lucha contra la comisión de delitos por parte de organizaciones, especialmente las corporaciones transnacionales ${ }^{19}$. Para este tipo de infracciones la represión por sí sola puede resultar poco eficaz para la sustancial ausencia, en la actualidad, de un sistema común que permita sancionar a las entidades que cometen delitos cuyos efectos van más allá de las fronteras nacionales ${ }^{20}$. El mejor remedio es, por lo tanto, ofrecer incentivos que les lleven a considerar más conveniente abstenerse de conductas ilícitas.

Por estas razones, el legislador italiano, con carácter innovador, exonera de responsabilidad cuando el órgano de administración haya adoptado y ejecutado con eficacia, antes de la comisión del delito, modelos de organización y gestión que incluyen las medidas

17 Por la relevancia práctica del "carrot and stick approach" en el sistema italiano de la responsabilidad de las entidades colectivas por los hechos delictivos, vid. C. De Maglie, Societas Delinquere potest? The Italian Solution, in AA.VV., Corporate Criminal Liability. Emergence, Convergence, and Risk, editado por M. PIETH, R. IvoRY, Springer, 2011, p. 262 s.

18 En el Informe del Gobierno al decreto (Relazione al d. lgs. 231/2001, cit.) el legislador italiano incorpora en su modelo el sistema estadounidense de los compliance programs. Para un examen, en perspectiva comparada, sobre el contenido de las Federal Sentencing Guidelines estadounidenses, que prescriben los criterios que deben seguir las personas jurídicas en la adopción de sus compliance programs, se puede hacer referencia a A.S. VALENZANo, Modelli organizzativi e organismo di vigilanza nel sistema della responsabilità dell'ente per fatti di reato, cit., p. 123 ss. También por la relevancia práctica del "carrot and stick approach" en las U.S. Federal Sentencing Guidelines, D. Izraeli, M.S. Schwartz, What Can We Learn From the U.S. Federal Sentencing Guidelines for Organizational Ethics?, European Institute for Business Ethics, in www.actrav.itcilo.org.

19 Sobre la importancia de los modelos de prevención en los grupos internacionales, P. BASTIA, I modelli organizzativi dei gruppi internazionali, in AA.VV., Reati e responsabilità degli enti. Guida al d. lgs. 8 giugno 2001, n. 231, segunda edición, editado por G. LATTANZI, Milano, 2010, p. 201 ss.

20 Sobre este tema, vid. S. MANACORDA, Appunti sulle potenzialità e i limiti di applicazione territoriale delle norme in materia di responsabilità degli enti, in AA.VV., Corporate criminal liability and compliance programs. First Colloquium, editado por 
de vigilancia y control idóneas para prevenir delitos de la "misma especie" (de la misma naturaleza) del que se ha cometido en particular, según una evaluación que debe realizarse ex ante con respecto al momento de la comisión del delito (porque de lo contrario ningun modelo de organización podría ser adecuado para prevenir) ${ }^{21}$.

En otras palabras, la responsabilidad de la entidad se puede excluir si se demuestra que la misma ha adoptado y ejecutado un modelo organizativo adecuado para prevenir delitos. Esto significa que la existencia de un nexo causal entre la "falta de organización" y el delito cometido no es por sí sola suficiente para la atribución del hecho delictivo a la entidad colectiva, sino que se requiere un nivel adicional en sede de atribución.

De acuerdo con parte de la doctrina italiana, este nivel adicional de atribución exige que, en ausencia de un adecuado modelo de organización, la conducta de la entidad se ha cometido fuera del área del "riesgo permitido" por el ordenamiento; área circunscrita por las normas contenidas en el propio modelo de prevención. En otras palabras, el nexo de causalidad entre la "falta de organización" y el delito en sí mismo no excluye la licitud de la conducta empresarial cuando dicha conducta, si bien arriesgada, debe considarse permitida por el ordenamiento porque se incluye en el área de "riesgo permitido"22.

Una importante confirmación de esta interpretación está en el mismo d. lgs. 231/2001 donde el art. 6 se refiere a la necesidad de que el modelo de organización y gestión sea "normalmente" adecuado para prevenir delitos de la "misma especie" (de la misma naturaleza) en relación a los que se han cometido, se bien no se refiere al delito específico realmente cometido. En otras palabras, la entidad no puede ser responsable por el hecho delictivo cometido si el modelo organizativo adoptado es idoneo para prevenir delitos "de la misma especie en el curso normal de los acontecimientos".

Sin embargo, la mayoría de la doctrina italiana relaciona este nivel adicional de la atribución a la llamada "culpa de organización" 23 , que debería corresponder al nivel "subjetivo" de la atribución, a pesar de todas las precauciones que ya hemos explicado porque se trata de personas jurídicas que no pueden tener un elemento subjetivo del delito idéntico al de las personas físicas.

Según esta interpretación, la “culpa de organización” está determinada por la

\footnotetext{
A. Fiorella, A.M. Stile, Nápoles, 2012, p. 409 ss.

21 En este sentido se expresa el art. 6 del d. lgs. n. 231/2001. Debe tenerse en cuenta que la opción del legislador italiano ha sido muy innovadora, pues en el sistema federal estadounidense, en el que si inspira la legislación italiana, la adopción de un programa de cumplimiento efectivo ("compliance program") constituye sólo una circunstancia atenuante de la pena a aplicar a la persona jurídica en la fase del sentencing, y no es una causa de exención de la responsabilidad como en el sistema italiano.

22 En la literatura, con esta interpretación, A. FIorella, Responsabilità da reato degli enti collettivi, cit., p. 5104; y, más en profundidad, A. Fiorella, A.S. Valenzano, Colpa dell'ente e accertamento. Sviluppi attuali in una prospettiva di diritto comparato, Roma, 2016, p. 56 ss.
}

23 Sobre el análisis y la referencia a las distintas posiciones en la doctrina, vid. A. BERNASCONI, Soggetti in posizione apicale 
violación de una norma de cuidado entendida como "culpa por incumplimiento"24 ("colpa per inosservanza") que se puede "reprochar" a la entidad colectiva y que consiste en la falta de adopción de una regla de precaución adecuada para prevenir delitos "de la misma especie" a los cometidos en concreto; norma de cuidado que se establece en los artículos 6 y 7 del d. lgs. n. 231/2001 o, en el campo de la salud y seguridad en el trabajo, en el art. 30 del d. lgs. n. $81 / 2008^{25}$.

\subsection{La carga de la prueba}

El decreto distingue la disciplina de la carga de la prueba, dependiendo de si el delito es cometido por un "ápice" (art. 6) o por una persona "subordinada" a la dirección o supervisión de uno de los líderes (art. 7), invirtiendo la carga en el primer caso, es decir, imponiendo a la entidad la carga de probar que estaba correctamente organizada para prevenir la comisión de delitos.

En otras palabras, cuando el autor del delito es un sujeto en posición apical, el decreto establece una presunción iuris tantum de responsabilidad contra la entidad, ya que en este caso se supone, en esencia, una "conexión estrecha" entre el delito que se ha cometido y la llamada "voluntad general" de la persona jurídica, porque el vertice debería representar a esta "voluntad general", que se expresa en las decisiones de organización y la gestión de la misma. Por supuesto, la entidad colectiva tiene la posibilidad de proporcionar pruebas para desvirtuar la presunción. Se trata, por tanto, de una presunción iuris tantum.

En mi opinión, debe criticarse la inversión de la carga de la prueba, porque está vulnera el principio de "presunción de inocencia" establecido por el art. 27 párrafo 2 de la Constitución italiana, en virtud del cual la carga de probar la culpabilidad del imputado es de la acusación ${ }^{26}$. Con esta norma, ya no es la acusación la que debe demostrar la "culpabilidad" de la entidad colectiva por el hecho delictivo, sino que la propia entidad colectiva tiene que defenderse proporcionando pruebas de su “correcta organización”, según los parámetros

\footnotetext{
e modelli di organizzazione dell'ente (Art. 6), in AA.VV., La responsabilità degli enti. Commento articolo per articolo al d. legisl. 8 giugno 2001, n. 231, editado por A. Presutti, A. Bernasconi, C. Fiorio, Padova, 2008. En la jurisprudencia, vid. Cass. pen., Sez. III, 7.6.2001, n. 28731, in www.rivista231.it; Cass. pen., Sez. VI, 18.2.2010, n. 27735, in www.rivista231.it.

24 La "culpa por incumplimiento" está establecida por el art. 43 del Código penal italiano, que la define como "el incumplimiento de las leyes, reglamentos, órdenes o disciplinas" ("l'inosservanza di leggi, regolamenti, ordini o discipline"). En este sentido se expresa el Informe del Gobierno al decreto (Relazione al d. lgs. 231/2001, cit.). Para un análisis del amplio debate doctrinal sobre este tema, también en perspectiva comparada, vid. A. Fiorella, A.S. Valenzano, Colpa dell'ente e accertamento. Sviluppi attuali in una prospettiva di diritto comparato, cit., p. 53 ss.; E. VIlLANI, Alle radici del concetto di 'colpa di organizzazione' nell'illecito dell'ente da reato, Napoli, 2016, p. 227 ss.

25 En el sentido de que la "culpa por incumplimiento" no puede consistir en el mero incumplimiento de una norma de cuidado general, siendo así necesario verificar la representatividad real del resultado, como lo ha reconocido la Corte Suprema (Cass. pen., Sez. Un., 29.5.2009, n. 22676), así como por la necesidad de identificar de forma precisa la norma de cuidado específica, en el sentido de estar diseñada específicamente para evitar el resultado: A. Fiorella, A.S. Valenzano, Colpa dell'ente e accertamento. Sviluppi attuali in una prospettiva di diritto comparato, cit., p. 53 ss.
}

26 Sin embargo, la jurisprudencia de la Corte de Casación se ha expreso en la dirección opuesta, asumiendo que no existe una 
establecidos en el art. 6 d. lgs. n. 231/2001.

La presunción de responsabilidad contra la entidad cesa, y vuelve al principio general de la carga de la prueba que incumbe a la acusación, cuando el autor es una persona "subordinada" a la dirección o supervisión de uno de los líderes (en posición "para-apical"), según el art. 7 del decreto. En otras palabras, en este caso, la entidad puede ser considerada responsable sólo si la fiscalía proporciona pruebas de que el delito ha "sido posible" gracias a la violación de las obligaciones de dirección o supervisión, es decir, la comisión del delito ha sido "causada" o, por lo menos, "facilitada" por la misma entidad.

La violación de estas obligaciones de dirección o supervisión está en cualquier caso excluida, y con ella también lo está la responsabilidad de la entidad por el delito, cuando ésta, antes de la comisión del delito, haya adoptado y ejecutado con eficacia modelos de organización, gestión y control idóneos para prevenir delitos "de la misma naturaleza" que los cometidos en caso particular.

\subsection{Los requisitos de los modelos de prevención de delitos}

Las condiciones que, en el caso de un delito cometido por un "ápice" (sujeto en posición "apical") deben probarse por la entidad para la exención de la responsabilidad por el hecho delictivo son las siguientes, de acuerdo con el art. 6 d. lgs. n. 231/2001:

a) el órgano de administración ha adoptado y ejecutado con eficacia, antes de la comisión del delito, un modelo de organización y gestión adecuado para prevenir delitos de la "misma especie" (de la misma naturaleza) que los cometidos en particular;

b) la supervisión del funcionamiento, de la eficacia y del cumplimiento del modelo de prevención implantado, así como su actualización, ha sido confiado a un organismo de la entidad colectiva con poderes autónomos de iniciativa y control ("organismo di vigilanza"27). En las personas jurídicas de pequeñas dimensiones, las funciones de supervisión pueden ser asumidas directamente por el órgano de administración;

c) no se ha producido una omisión o un ejercicio insuficiente de sus funciones de supervisión por parte del mismo organismo ("organismo di vigilanza");

d) los autores individuales han cometido el delito eludiendo fraudulentamente los modelos de organización y gestión (a este asunto me referiré en breve).

inversión de la carga de la prueba. Vid. Cass. pen., Sez. VI, 16 luglio 2010, n. 27735, cit.

27 Sobre un análisis de las funciones y características del "organismo di vigilanza", vid. A.S. VALENZANo, Modelli organizzativi e organismo di vigilanza nel sistema della responsabilità dell'ente per fatti di reato, cit., p. 39 ss. Más recientemente, E. MezzetTi, Spunti di riflessione su composizione e requisiti dell'Organismo di Vigilanza ai sensi del d. lgs. n. 231 del 2001, in AA.VV., Corporate criminal liability and compliance programs. First Colloquium, p. 421 ss. 
Por lo que respecta al contenido del modelo de prevención de delitos, el legislador distingue de nuevo en función de si se trata de un delito cometido por un "ápice” o por una persona "subordinada" a la dirección o supervisión de uno de los líderes.

Con referencia al primer caso, es decir, el delito se atribuye al "ápice”, el decreto establece que, teniendo en cuenta el sistema de delegación de poderes, el modelo preventivo debe cumplir con los siguientes requisito ${ }^{28}$ :

a) identificar las actividades en cuyo ámbito puedan ser cometidos los delitos que deben prevenirse ("mappatura del rischio");

b) establecer los protocolos (o procedimientos) específicos para planificar la formación y la ejecución de las decisiones de la persona jurídica con relación a los delitos que deben prevenirse;

c) identificar los modelos de gestión de los recursos financieros adecuados para impedir la comisión de los delitos;

d) imponer obligaciones de información al organismo encargado de vigilar el funcionamiento y la observancia del modelo de prevención;

e) establecer un sistema disciplinario que sancione adecuadamente el incumplimiento de las medidas establecidas en el modelo de prevención.

El legislador también ha introducido una especie de mecanismo de "acuerdo" sobre el contenido del modelo de organización y gestión, estableciendo que el mismo puede adoptarse sobre la base de "códigos de conducta" ("codici di comportamento"), elaborados por las asociaciones representativas de las empresas ("linee-guida") y notificados al Ministerio de Justicia, que puede formular observaciones sobre la "idoneidad" de los modelos de prevención de delitos.

Se trata, en nuestra opinión, de mecanismo de "acuerdo" sobre el contenido del modelo de prevención demasiado "tímido", entre las autoridades públicas y las entidaded privadas, que sin duda podría tener un mayor impacto en la práctica si el legislador sin llegar a establecer mecanismos de "consulta" pura, hubiese previsto mecanismos más eficaces para garantizar la seguridad jurídica a las entidades colectivas, para así saber con certeza qué es necesario para considerar idóneo un modelo de organización de prevención de delitos ${ }^{29}$.

Con referencia al segundo caso, es decir, cuando el delito se atribuye a una persona "subordinada" a la dirección o supervisión de uno de los ápices, el legislador ha establecido

28 Sobre la necesidad de que el modelo de organización constituya una especie de "tarjeta de identidad" de la entidad colectiva y sobre los pasos instrumentales de su realización, vid. C. PIERGALlnin, Paradigmatica dell'autocontrollo penale (dalla funzione alla struttura del "modello organizzativo" ex D. Lgs. 231/2001), in CSM, Le tipologie di colpa penale tra teoria e prassi, Roma, 28 - 30 marzo 2011, in www.csm.it, p. 11 ss.

29 Sobre esta cuestión, infra, n. 11. 
elementos del modelo de prevención que parecen más simples (menos estrictos), indicando que es suficiente que el modelo de organización, gestión y control contenga las medidas que, de acuerdo con la naturaleza y el tamaño de la organización, así como el tipo de actividades que se llevan a cabo, garanticen el desarrollo de su actividad conforme a la ley y permitan la detección rápida y prevención de situaciones de riesgo (según el art. 7 d. lgs. n. 231/2001).

La aplicación eficaz de este modelo de prevención requiere, en cualquier caso, una verificación periódica del mismo y de su eventual modificación cuando se pongan de manifiesto infracciones relevantes de sus disposiciones, o cuando se produzcan cambios en la organización o en la actividad desarrollada que los hagan necesarios; y de un sistema disciplinario que sancione adecuadamente las infracciones de las medidas de control y organización establecidas en el modelo de prevención.

\section{La elusión fraudulenta del modelo de organización y gestión}

El quinto y último criterio de atribución de la responsabilidad a la entidad colectiva se establece en el art. 6 únicamente para el caso en que el delito haya sido cometido por una persona en posición "apical". Dicho precepto se refiere a la elusión no fraudulenta del modelo organizativo por parte del autor del delito como elemento de conexión necesario entre el delito y la misma entidad. En otras palabras, en este caso la persona jurídica puede ser responsable solo si el delito ha sido cometido a través de una elusión no fraudulenta del modelo organizativo por parte del "ápice”.

A contrario, el decreto establece que, cuando la organización está adecuadamente organizada, ésta puede quedar exenta de responsabilidad si prueba que el delito fue cometido por el líder a través de una "elusión fraudulenta" de las reglas de conducta establecidas en el modelo de organización ("elusione fraudolenta del modello"). Esta causa de exclusión de responsabilidad se fundamenta en el hecho de que el fraude del líder no se puede atribuir automáticamente a una entidad correctamente organizada, porque de lo contrario se violaría el principio de "personalidad de la responsabilidad penal" 30 .

En resumen, para evaluar si la "actitud corporativa" ha contribuido realmente a las actividades ilegales, el legislador italiano requiere una investigación adicional sobre las posibles políticas ilegales perseguidas en secreto por la entidad a pesar de la presencia de una organización teóricamente adecuada para prevenir delitos ${ }^{31}$.

30 En este caso, se habla de un "administrador infiel". En este sentido, vid. el Informe del Gobierno al decreto (Relazione al d. lgs. n. 231/2001, cit.).

31 En la jurisprudencia, una persona jurídica ha sido declarada inocente teniendo en cuenta que los líderes cometieron el delito "incumpliendo" de manera fraudulenta el modelo organizativo adoptado por la misma: Trib. Milano, 17.11.2009, in Le società, 2010, 


\section{El catálogo de delitos previstos en el decreto legislativo n. 231/2001}

Las entidades colectivas pueden ser responsables únicamente con respecto a los delitos incluidos en el catálogo del decreto legislativo n. 231/2001, siempre que se trate de delitos cometidos por un miembro individual de la entidad y en su interés.

El catálogo debe entenderse como exhaustivo, es decir que no es extensible a otros delitos, pues de ser así se estaría llevando a cabo una interpretación analógica vetada en materia penal. El catálogo, por tanto, debe entenderse como un numerus clausus (artículos de 24 a 25 duodecies del d. lgs. n. 231/2001).

Con un planteamiento inicialmente cauto por parte del legislador italiano que tendía a delimitar la responsabilidad de las entidades colectivas sólo a los delitos contra la administración pública (incluida la corrupción), numerosas reformas han ampliado enormemente la lista de los delitos por los que la entidad puede ser responsable, si bien aún no es equiparable al número de delitos susceptibles de ser cometidos por una persona física, tal y como ocurre en Francia ${ }^{32}$.

Nuestro legislador ha ampliado expresamente la responsabilidad de las entidades colectivas a los delitos societarios, los delitos contra la fe pública, los delitos informáticos, los delitos de abuso del mercado, los crímenes contra la industria y el comercio, los delitos de receptación y reciclaje, algunos delitos contra las personas, así como el homicidio y lesiones culposas en violación de las normas relativas a la protección de la salud y seguridad en el trabajo, los delitos de delincuencia organizada, también los de carácter transnacional, los delitos de terrorismo o subversión del orden democrático, los crímenes en relación a la infracción de los derechos de autor, el delito de incitación a no declarar o de falso testimonio ante un tribunal, los delitos medioambientales y, en última instancia, el delito de uso de nacionales de terceros países cuya estancia es ilegal. También hay un proyecto de reforma para introducir los delitos fiscales.

Desde otro punto de vista, es evidente que la continua ampliación de la esfera de la responsabilidad de las entidades por la comisión de delitos produce el efecto de aumentar también las áreas de riesgo de comisión de delitos que deben ser vigiladas por el modelo prevención de delitos que, también por esta razón, debe ser actualizado constantemente.

\section{El abanico de sanciones en el caso de entidades colectivas}

p. 476, con comentario de C.E. PALIERo, Responsabilità dell'ente e cause di esclusione della colpevolezza: decisione «lassista»o interpretazione costituzionalmente orientata?; sobre el mismo caso, Corte App. Milano, 18.6.2012, in www.rivista231.it.

32 En particular, se trata de la ley n. 204/2004 ("Loi Perben II”), que entró en vigor el 31.12.2005, y que ha modificado el artículo 121-2 del Código penal francés extendiendo la responsabilidad penal de las personas jurídicas a todo tipo de delitos. Sobre el significado de la modificación de la ley, en la doctrina francesa, vid. E. PIRE, La responsabilité pénale des personnes morales: difficultés de droit transitoire, in Recueil Dalloz, 2004, p. 1650 ss. 
El d. lgs. n. 231/2001 contiene un amplio abanico de sanciones que se pueden aplicar a la entidad considerada responsable de un hecho delictivo y, dentro de ciertos límites, a la misma entidad en la fase de instrucción, es decir, como medida cautelar de forma independiente a la condena penal.

El régimen sancionador es, por así decirlo, "bipartito" en el sentido de que se preven las penas de la multa así como sanciones privativas de otros derechos.

Las penas aplicables a las personas jurídicas son las siguientes:

a) Multa por cuotas;

b) Suspensión ("interdizione”) de sus actividades. Esta podrá ser temporal y en algunos casos definitiva;

c) Suspensión o revocación ("sospensione o revoca") de autorizaciones, licencias o concesiones funcionales para la comisión del delito;

d) Prohibición de contratar ("divieto di contrattare") con la administración pública, a excepción de la prestación de un servicio público;

e) Inhabilitación ("esclusione e revoca") para obtener subvenciones y ayudas públicas y la posible revocación de las ya concedidas;

f) Prohibición de publicitar ("divieto di pubblicizzare”) bienes o servicios;

g) Decomiso;

h) Publicación de la sentencia.

Las sanciones privativas de derechos deben referirse a la "actividad específica" sobre la que recae la infracción cometida por la entidad, la llamada "unidad organizativa"33 (en virtud del art. 14, párrafo 1, y art. 69, párrafo 2, d. lgs. 231/2001). El juez determina el tipo y la duración de la sanción teniendo en cuenta también la idoneidad de las sanciones para prevenir delitos de la misma naturaleza de los cometidos ${ }^{34}$.

Es además interesante resaltar que las sanciones privativas de derechos no pueden aplicarse cuando la entidad colectiva haya reparado las consecuencias del delito antes de la declaración de apertura del juicio (art. 17 d. lgs. n. 231/2001). A este respecto, se entiende por reparación:

- haber procedido a reparar íntegramente el daño causado por el delito o a eliminar las consecuencias nocivas o peligrosas del delito, o haber tomado medidas efectivas en esta dirección;

- haber eliminado las “deficiencias organizativas" ("carenze organizzative”) que

33 En la jurisprudencia, en este sentido, Cass. pen., Sez. VI, 28.9.2011, n. 43108.

34 En el sentido de que las sanciones deben imponerse a las entidades colectivas garantizando los principios de economía y proporción de las sanciones, se expresa el Informe del Gobierno al decreto (Relazione al d. lgs. n. 231/2001, cit.) 
llevaron al delito a través de la adopción y ejecución de modelos de organización adecuados para prevenir delitos "de la misma naturaleza" de los cometidos, a través del llamado "modelo de reparación" ("modello riparatore") o post factum; - haber puesto a disposición el beneficio obtenido para fines de decomiso.

\section{Conclusiones y perspectivas de reforma}

En conclusión, la disciplina italiana sobre la responsabilidad de las entidades colectivas por hechos delictivos, aunque muy valiosa, ya que subraya el "defecto de organización" y la "culpa de organización" a los efectos de la atribución de la responsabilidad a la persona jurídica, muestra, sin embargo, algunos aspectos problemáticos tras más de diez años desde su entrada en vigor.

En primer lugar, resulta problemática la inversión de la carga de la prueba que incumbe a la entidad en el caso de que el delito haya sido cometido por un sujeto en posición "apical”35.

En segundo lugar, desconcierta la casi total ausencia de reconocimiento de la “idoneidad" de los modelos de organización por la jurisprudencia italiana ${ }^{36}$, inclinada sustancialmente a reconocer la responsabilidad de las entidades por los hechos delictivos y a declarar los modelos en concreto adoptados como "no adecuados"37.

Esta situación produce una sustancial ausencia de seguridad jurídica para las empresas, aunque, como ya se mencionó, el d. lgs. 231/2001 (art. 6, párrafo 3) ha establecido una especie de mecanismo de "acuerdo" sobre el contenido del modelo de organización y gestión, entre las entidades privadas y las autoridades públicas (en concreto, el Ministerio de Justicia), ya que los modelos de prevención pueden adoptarse sobre la base de "códigos de conducta" ("codici di comportamento" o "linee-guida") elaborados por las asociaciones representativas de las empresas.

Sin embargo, este mecanismo no ofrece ninguna "garantía de idoneidad" del modelo de organización para prevenir delitos, ya que no se refiere al modelo específico adoptado por una entidad colectiva, sino que se refiere únicamente a los "códigos de conducta" más genéricos ("linee-guida"), que sirven como punto de referencia a seguir por cada entidad en la elaboración de su modelo más específico, que debe ser construido en concreto sobre la organización en particular y sobre las actividades individuales ejercitadas por cada persona jurídica a modo de "traje de chaqueta" ( "abito su misura”).

35 Sobre este tema, supra, n. 7.1.

36 Para un análisis de la jurisprudencia, también sobre el modelo organizativo adoptado post factum, E. VILLANI, Corporate liability 'ex crimine' and compliance programs in Italy, in AA.VV., Corporate criminal liability and compliance programs, Vol. I, Liability 'ex crimine' of legal entities in Member States, cit., p. 19.

37 Para un análisis más detallado de los problemas, sobre todo de carácter práctico, que han surgido en Italia, vid. AA.VV., $L a$ responsabilità da reato degli enti collettivi: a dieci anni dal d.lgs. n. 231/2001. Problemi applicativi e prospettive di riforma, cit. 
En otras palabras, el hecho de que una entidad colectiva haya construido su modelo de organización y gestión específico implementando las "linee-guida" aprobadas por el Ministerio de Justicia no ofrece en sí mismo ninguna "garantía" para la entidad sobre la “idoneidad" del modelo de organización para prevenir delitos, es decir para la exención de su responsabilidad, ya que el juicio en concreto sobre la idoneidad para prevenir los delitos de cada modelo adoptado es competencia de los tribunales penales que, en Italia, tienden a no reconocer la idoneidad del modelo cuando se ha cometido un un delito ${ }^{38}$.

En nuestra opinión, sería posible superar este estado de inseguridad jurídica para las entidades mediante el reconocimiento de mecanismos de "certificación de la idoneidad" para la prevención de delitos de "cada" modelo de organización en concreto adoptado por la persona jurídica, como ya se ha propuesto en un reciente proyecto de reforma del d. $\operatorname{lgs}$. $231 / 2001^{39}$ y como, por otra parte, ya se prevé en el caso chileno ${ }^{40}$.

De acuerdo con el citado proyecto de reforma italiano, una entidad que haya adoptado un modelo de organización cuya idoneidad para prevenir delitos esté certificada por una tercera entidad certificadora, externa a la persona jurídica, estará exenta de responsabilidad por el delito cometido, a excepción de los casos donde se haya producido una "señal de alarma grave", porque hayan surgido violaciones importantes de las reglas que pongan de manifiesto el "defecto de organización" que hubiese causado el delito imputado, ya que un “defecto de organización" obliga a la entidad a intervenir para poner remedio a las "carencias organizativas" $"$.

El mecanismo propuesto de "certificación" de la idoneidad del modelo adoptado para prevenir delitos podría garantizar en la práctica el principio fundamental de la "personalidad de la responsabilidad" de la persona jurídica por los hechos delictivos, ya que ésta sería responsable sólo por un hecho "propio", que es "personal” y "culpable", es decir, sólo por su propio "defecto de organización" que se le puede "reprochar", en ausencia del cual la misma entidad estaría exenta de responsabilidad por el delito cometido por la persona física.

38 Sobre el debate que se ha desarrollado en este tema en la doctrina y la jurisprudencia, vid. G. FIDELBo, L'accertamento dell'idoneità del modello organizzativo in sede giuziale, in AA.VV., La responsabilità da reato degli enti collettivi: a dieci anni dal d.lgs. n. 231/2001. Problemi applicativi e prospettive di riforma, cit., p. 173 ss.

39 La reciente propuesta de reforma del d. lgs. n. 231/2001, que ha sido preparada y presentada por el AREL el 7.7.2010, propone la introducción de un nuevo art. 7-bis en el d. lgs. n. 231/2001, que establece la "certificación sobre la idoneidad del modelo de organización para prevenir delitos" adoptado por la entidad colectiva: Schema di disegno di legge di modifica del d.lgs. 8 giugno 2001 n. 231, in AA.VV., La responsabilità amministrativa degli enti. Progetto di modifica D.Lgs. 231/2001, editado por R. PINZA, Roma, 2010, p. 9 s.

40 La disciplina chilena de la responsabilidad penal de las personas jurídicas, claramente inspirada en la legislación italiana, incluye mecanismos para la certificación de la idoneidad del modelo de organización para la prevención de delitos. Vid. Ley n. 20.393 de 2.12.2009, y especialmente el artículo 4.

41 Para un análisis sobre este tema, así como sobre el contenido del proyecto de reforma antes mencionado, vid. A. FioRELLA, Responsabilità individuale e responsabilità degli enti negli infortuni sul lavoro, cit., p. 274 ss. 


\section{Bibliografía}

AA.VV., Corporate criminal liability and compliance programs, Vol. I, Liability 'ex crimine' of legal entities in Member States, editado por A. FIORELLA, Nápoles, 2012.

AA.VV., La responsabilità da reato degli enti collettivi: a dieci anni dal d.lgs. n. 231/2001. Problemi applicativi e prospettive di riforma, editado por V. MONGILLO, A.M. STILE, G. STILE, Nápoles, 2013.

AMARELLI, G. Mito giuridico ed evoluzione della realtà: il crollo del principio societas delinquere non potest, in Riv. trim. dir. pen. econ., 2003.

BASTIA, P. I modelli organizzativi dei gruppi internazionali, in AA.VV., Reati e responsabilità degli enti. Guida al d. lgs. 8 giugno 2001, n. 231, segunda edición, editado por G. LATTANZI, Milano, 2010.

BERNASCONI, A. Soggetti in posizione apicale e modelli di organizzazione dell'ente (Art. 6), in AA.VV., La responsabilità degli enti. Commento articolo per articolo al d. legisl. 8 giugno 2001, n. 231, editado por A. PRESUTTI, A. BERNASCONI, C. FIORIO, Padova, 2008.

CARMONA, A. Premesse a un corso di diritto penale dell'economia, Padova, 2002.

DE MAGLIE, C. Societas Delinquere potest? The Italian Solution, in AA.VV., Corporate Criminal Liability. Emergence, Convergence, and Risk, editado por M. PIETH, R. IVORY, Springer, 2011.

DE SIMONE, G. La responsabilità da reato della societas nell'ottica dei principi costituzionali, in AA.VV., La responsabilità da reato degli enti collettivi: a dieci anni dal d.lgs. n. 231/2001.

DE VERO, G. La responsabilità penale delle persone giuridiche, in Trattato di diritto penale, diretto da C.F. GROSSO, T. PADOVANI, A. PAGLIARO, Milano, 2008.

DI GIOVINE, O. La responsabilità degli enti: lineamenti di un nuovo modello di illecito punitivo, in AA.VV., Diritto e impresa: un rapporto controverso, editado por A. MANNA, Milán, 2004.

FIDELBO, G. L'accertamento dell'idoneità del modello organizzativo in sede giuziale, in AA.VV., La responsabilità da reato degli enti collettivi: a dieci anni dal d.lgs. n. 231/2001.

FIORELLA, A. Responsabilità da reato degli enti collettivi, in Dizionario di diritto pubblico, vol. V, dirigido por S. Cassese, Milano, 2006. 
; VALENZANO, A.S. Colpa dell'ente e accertamento. Sviluppi attuali in una prospettiva di diritto comparato, Roma, 2016

FLORA, G. Le sanzioni punitive nei confronti delle persone giuridiche: un esempio di «metamorfosi» della sanzione penale?, in Dir. pen. proc., 2003.

FORTI, G. Sulla definizione della colpa nel progetto di riforma del codice penale, in C. DE MAGLIE, S. SEMINARA, La riforma del codice penale. La parte generale, Actas de la Conferencia de Pavia, 10-12 maggio 2001, Milán, 2002.

GOBERT, J.; PUNCH, M. Rethinking Corporate Crime, Cambridge, 2003.

.; MUGNAI, E. Coping with Corporate Criminality - Some Lessons from Italy, in Criminal Law Review, 2002.

GUERRINI, R. La responsabilità da reato degli enti. Sanzioni e loro natura, Milano, 2006.

IZRAELI, D. ; SCHWARTZ, M.S. What Can We Learn From the U.S. Federal Sentencing Guidelines for Organizational Ethics?, European Institute for Business Ethics, in www. actrav.itcilo.org.

MAIELLO, V. La natura (formalmente amministrativa, ma sostanzialmente penale) della responsabilità degli enti nel d. lgs. n. 231/2001: una «truffa delle etichette» davvero innocua?, in Riv. trim. dir. pen. econ., 2002.

MANACORDA, S. Appunti sulle potenzialità e i limiti di applicazione territoriale delle norme in materia di responsabilità degli enti, in AA.VV., Corporate criminal liability and compliance programs. First Colloquium, editado por A. FIORELLA, A.M. STILE, Nápoles, 2012.

MANNA, A. La c.d. responsabilità amministrativa delle persone giuridiche: il punto di vista del penalista, in Cass. pen., 2003.

MASSI, S. 'Veste formale'e 'corpo organizzativo'nella definizione del soggetto responsabile per l'illecito da reato, Nápoles, 2012.

MASUCCI, M. Infedeltà patrimoniale e offesa al patrimonio nella disciplina penale dei gruppi di società, Nápoles, 2006.

MEZZETTI, E. Spunti di riflessione su composizione e requisiti dell'Organismo di Vigilanza ai sensi del d. lgs. n. 231 del 2001, in AA.VV., Corporate criminal liability and compliance programs. First Colloquium, p. 421 ss. 
MUSCO, E. Le imprese a scuola di responsabilità tra pene pecuniarie e misure interdittive, in Dir. e giust., 2001.

NIETO MARTín, A. El artículo 31 bis del Código Penal y las reformas sin estreno, in Diario la ley, 2014, n. 8248.

PADOVANI, T. Il nome dei principi e il principio dei nomi: la responsabilità "amministrativa" delle persone giuridiche, in AA.VV., La responsabilità degli enti: un nuovo modello di giustizia “punitiva”, editado por G. DE FRANCESCO, Torino, 2004.

PALIERO, C.E. Il d. lgs. n. 231/2001: da ora in poi, societas delinquere (et puniri) potest, in Corr. Giur., 2001, n. 7.

PATRONO, P. Verso la soggettività penale di società ed enti, in Riv. trim. dir. pen. econ., 2002.

PIERGALLINI, C. Paradigmatica dell'autocontrollo penale (dalla funzione alla struttura del “modello organizzativo" ex D. Lgs. 231/2001), in CSM, Le tipologie di colpa penale tra teoria e prassi, Roma, 28 - 30 marzo 2011, in www.csm.it, p. 11 ss.

PIRE, E. La responsabilité pénale des personnes morales: difficultés de droit transitoire, in Recueil Dalloz, 2004.

PISANI, N. La struttura dell'illecito e i criteri di imputazione, in AA.VV., La responsabilità da reato degli enti, vol. X, editado por A. D'AVIRRO e A. DI AMATO, Padova, 2009.

PULITANÒ, D. Responsabilità amministrativa per i reati delle persone giuridiche, in Enc. dir., 2002.

SELVAGGI, N. L'interesse dell'ente collettivo quale criterio di ascrizione della responsabilità da reato, Nápoles, 2006.

STORTONI, L.; TASSINARI, D. La responsabilità degli enti: quale natura? Quali soggetti?, in Indice pen., 2006.

VALENZANO, A.S. Modelli organizzativi e organismo di vigilanza nel sistema della responsabilità dell'ente per fatti di reato, Roma, 2007.

. 'Triggering persons': the role of the offender in 'ex crimine' liability of legal entities. A comparative study on Member States'legal systems, in AA.VV., Corporate criminal liability and compliance programs, Vol. II, Towards a common model in the European Union, editado por A. FIORELLA, Nápoles, 2012. 
. Responsabilità degli enti e criminalità transnazionale anche nella prospettiva dei rapporti tra l'Unione Europea e le Nazioni Unite, in AA.VV., Rapporti tra Unione Europea e organizzazioni internazionali, editado por E. PFÖSTL, Roma, 2009.

VILLANI, E. Alle radici del concetto di 'colpa di organizzazione' nell'illecito dell'ente da reato, Napoli, 2016.

\section{SOBRE A AUTORA:}

\section{Anna Salvina Valenzano}

Doctora en Derecho Penal y Abogada, Investigadora en Universidad Sapienza de Roma (Italia), desde el año 2014, Docente de la Escuela de especialización para las profesiones legales de la misma Universidad, Docente encargada del módulo denominado "Responsabilidad administrativa por delitos y compliance programs ex d. lgs. n. 231/2001" del curso de alta formación de la Universidad Sapienza "Organización de la empresa y responsabilidad penal en la tutela de la salud y la seguridad en el trabajo".

Ha participado en diversos proyectos de investigación, nacionales e internacionales, entre los que destaca la investigación tri-anual financiada por la Unión Europea, titulada "Corporate Criminal Liability and Compliance

Programs", llevada a cabo entre los años 2009-2012.

Autora de las obras "Modelos organizativos y organismo de vigilancia en el sistema de responsabilidad del ente por hechos delictivos", Roma, 2007; "Culpa del ente y verificación. Desarrollos actuales en una perspectiva de derecho comparado" (Co-autor A. Fiorella), Roma, 2016; así como de numerosos ensayos en italiano, inglés y español en la materia de la responsabilidad de la persona jurídica por delitos y, en general, de derecho penal económico.

Por último, ha participado como relatora en numerosos convenios, tanto a nivel nacional como internacional.

\section{DESC

